\title{
Detection of Tumor Objects from MRI Brain Images using Thresholding Segmentation
}

\author{
Sathish Kumar K, Venkatesh S,
}

\begin{abstract}
Nowadays identification of brain tumor is a critical and challenging work in the research field. We describe the detection of brain tumor by using thresholding segmentation. Segmentation of brain tumor in MRI is developing research works. It is used to identify the tumor shape, size, and exact location. Our proposed system has three stages to detect the brain tumor in MRI. Thresholding technique is developed to detect the brain tumor in MRI. The first stage is to collection MRI data from the web database. The second stage for pre-processing and finally post-processing. The proposed system is less time consuming while compared to other techniques and the medical experts are also easily identify the exact tumor location.
\end{abstract}

Keywords : MRI (Magnetic Resonance Imaging), Brain tumor, Thresholding Segmentation, Edge Detection.

\section{INTRODUCTION}

The brain is a most important organ in our body. It is the largest also. The brain has more than 100 billion nerves system and it is linked with trillions of connections called synapsis.

The brain is separated into four types of lobes. The brain lobes are frontal, parietal, temporal and occipital. An analytical approach, decision and motor function which is controlled by frontal lobe. Feeling, handwriting and position which is controlled by parietal lobe. Memorial and hearing which is managed by temporal lobe. Finally, the occipital lobe managed the brain's optical system. Brian tumor is a second leading disease. Mostly it affects children and young adults. Based on Central Brain Tumor Registry of the United States (CBTRUS), at end of 2011, the survey said that there are 64,530 people having a primary brain tumor and more than 600,000 people currently live with the tumor.

Normally, growing of any abnormal tissue in the brain which is named brain tumor. Usually, it caused by more pressure on brain tissue. To finding out the tumor from MRI brain images are very important and time-consuming also. It has some difficulty by performed manually. Medical experts are spent a large amount of time in radiologists for detection of the tumor from brain tissues.

Types of Tumor:

Three types of tumor commonly occur in young people. There are benign, Pre-malignant and malignant.A benign brain tumor is not affected other organs. It can be easily removable and it doesn't appear again once

Revised Manuscript Received on December 16, 2019.

* Correspondence Author

Sathish Kumar K *, School of Electrical Engineering, VIT University, Vellore, India. Email: kansathh21@yahoo.co.in

Venkatesh S, School of Electrical Engineering, VIT University, Vellore, India. Email: srinivasan.venkatesh@vit.ac.in
treated.Pre-malignant is a pre-cancerous stage. It causes cancer if not cured.Malignant is a most critical stage and it develops faster than the benign tumor.

There are many algorithms were developed in the last few years for brain tumor detection. But it has some drawbacks like not properly extraction in the detection of the image.

In this paper, we describe thresholding segmentation are used to find tumor objects in MRI brain image. Our proposed system detect the tumor in three steps. There are data collection, Pre-processing, and Post-processing. The MRI brain data was collected from the web database. Preprocessing is the one of the basic technique to enhance the image quality. In image analysis which is the primary step. It is removed unwanted distortion or noise in MR brain image. Thresholding segmentation is used to extract the tumor objects from the MR brain images.

Literature survey:

An efficient detection of the brain tumor region by Fuzzy $\mathrm{C}$-means clustering and histogram in the cerebral image as referred in [5]. In this scheme, they used the histogram equalization to calculate the intensity values of the gray level images. In order to reduce the dimensionality of the wavelet co-efficient the decomposition of images has done by principle component analysis. The k-means clustering technique is referred [3] to track the tumor objects in the Magnetic Resonance (MRI) brain images by color based segmentation method. A color segmentation with K-means is to convert grayscale MRI into a color MRI and then separate the position of tumor objects. The threshold and Watershed segmentation is very simple and popular [10]. Adaptive brain tumor detection is proposed [11] using K-Means segmentation with preprocessing of the image. Which is denoising by the Median filter. Therefore in order to obtain more detailed information of tumor region they are using object labeling. SVM is used to make adaptive system. There they proposed for detection and identification of brain tumor [4] is referred using a Neural Network segmentation relies on processing. In this paper, they have describes an Algorithm for identifying the shape of the tumor in brain MRI images. [8] In these paper they have used computer-aided method for tumor detection based on two algorithms also it reduces analysis time. The tumor is detected from the MRI image and determined tumor's position.

\section{PROCEDURE AND METHODOLOGY}

MRI brain images are used here to detect the brain tumor in MATLAB. The following flow diagram shows the overall process of brain tumor detection by using thresholding segmentation.

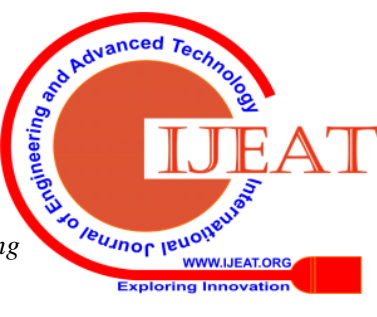




\section{Detection of Tumor Objects from MRI Brain Images using Thresholding Segmentation}

Algorithm for brain tumor detection:

1. Input the MRI brain image

2. Convert the MRI color image into grayscale image

3. Apply filtering for noise removal

4. Apply histogram equalization for image enhancement

5. Applying border removal for removing unnecessary portion in MRI

6. Extract the tumor object from MRI by using thresholding segmentation

7. Finally, boundary extraction is done by canny edge detector

\section{PROCEDURE:}

\section{A. Image Data Collection:}

The MRI brain image data was collected from the database. Normal and abnormal brain MRI images are downloaded from the web database.

The following Figure-1 shows that the normal and abnormal MRI brain image.
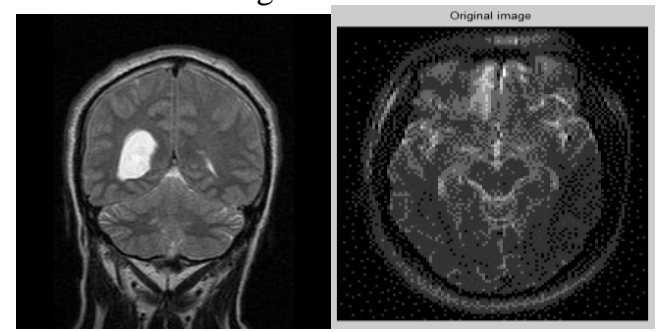

Fig. 1. Abnormal and Normal Brain MRI Image

\section{B. Pre-processing}

The main goal of this Pre-processing is to remove the undesired components and reducing noise for enhance the image quality and that is useful for further processing. The pre-processing is done by five steps. There are,

1. Grayscale conversion

2. Filtering

3. Image enhancement

4. Border removal

5. Normalization

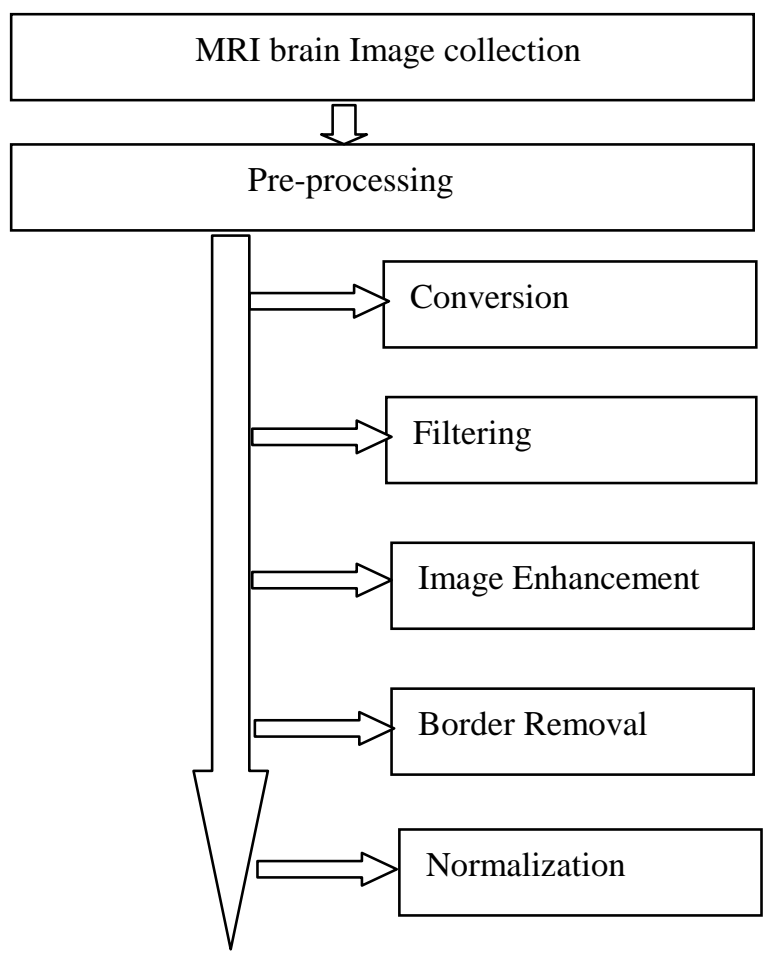

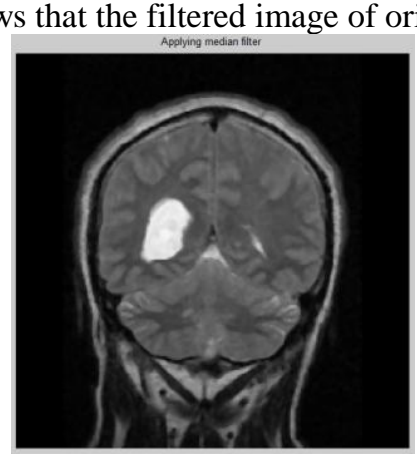

Fig. 3. Median filtered image

\section{E. Image Enhancement}

Image enhancing technique are used to improve the quality of the image. The basic enhancement of the MRI is the contrast enhancement. Here, we are performing histogram equalization to enhancing the MRI images. It depends on the frequency techniques otherwise spatial techniques. Boundaries and edges of MRI images are enhanced by image enhancement for analysis.Histogram Equalization is a method to modify the intensity of the image by defining the value of pixels. The following figure shows that the image enhancement of filtered image.

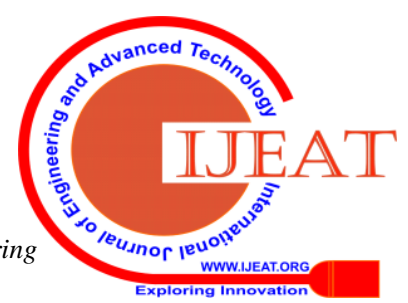




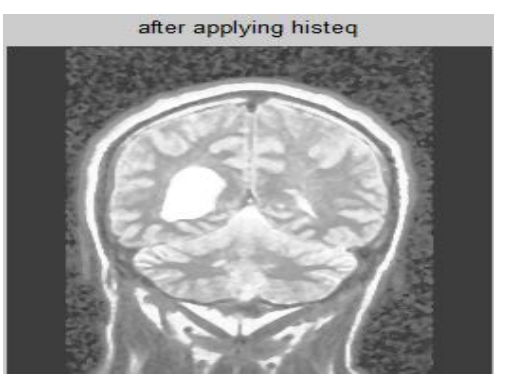

Fig. 4. Histogram Equalization of MRI

\section{BORDER REMOVAL}

Border removal which is a technique used to remove the unnecessary border around the MRI objects. These borders occur while applying histogram equalization. This border removal is done by using MATLAB inbuilt function called imclearborder.

The following figure shows that the border removal image of after applying histogram equalization image.

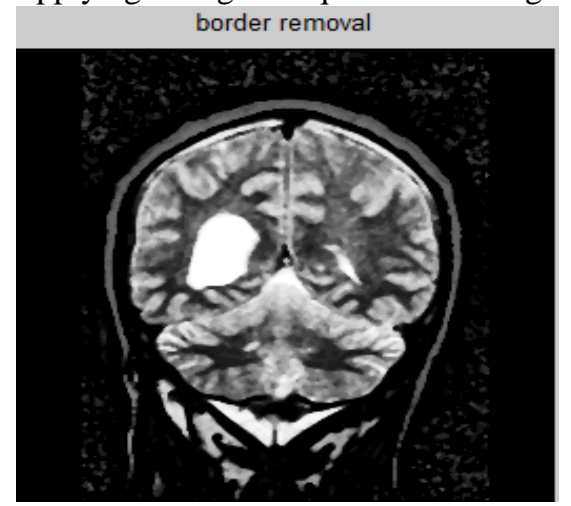

Fig. 5. Border Removal image of MRI brain image

\section{NORMALIZATION}

Normalization is a technique to modify the intensity values of the image. This is done by the following equation.

$$
I=(I-\operatorname{Min}) \frac{\text { newMax }- \text { newMin }}{\operatorname{Max}-\operatorname{Min}}+\text { newMin }
$$

From above equation,

\section{Max=255 and Min=0,}

newMax and newMin are the intensity values of the image.

Basically, normalization changes the intensity values, if the intensity value is less than 0 or greater than the 255 , it will normalize within the intensity value 0 to 255 .

Post-processing:

Post-processing is the third stage of our proposed technique. There are two processes under this processing. The processes are a segmentation and edge detection.

Segmentation:

Image segmentation which is the process of dividing the image into different segments. The fundamental of the segmentation is to changing the simplified image into a meaningful image for easily analyzing purpose. Basically, it is used to identify the boundaries and objects of MRI brain image.

\section{THRESHOLDING SEGMENTATION}

Thresholding segmentation is the simplest method in image segmentation technique. Firstly, the grayscale image is transformed into binary image format. The main aim is to choosing the threshold value. The threshold value is compared with the all pixel value of MRI image. If the threshold value is greater than the pixel intensity value that will be marked as white or if lower than the threshold value that will be marked as a black. Finally, the high-intensity values of MRI image are extracted and that is called tumor region.

The following figure shows that the tumor extracted image from the MRI brain image.

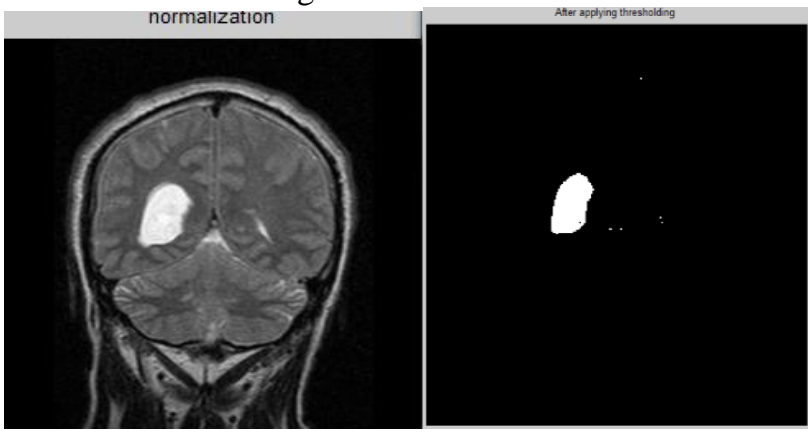

Fig. 6. Tumor Extracted Brain MRI image.

From above figure, we can identify the exact location of a brain tumor.

\section{A. Edge Detection}

Here, a canny edge detector is used for boundary extraction. Because it is efficient detector from another edge detector.

The following figure shows that the boundary extracted from the MRI by using canny edge detection.

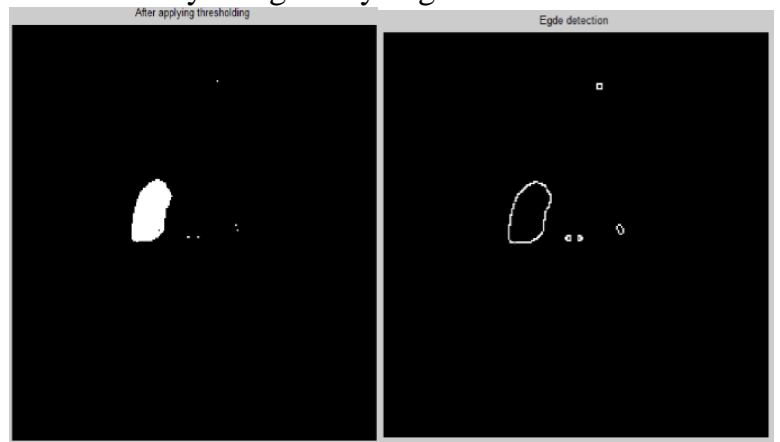

Fig. 7. Canny Edge Detection image of MRI

\section{RESULT AND DISCUSSION}

The following figures show that the image analysis normal and abnormal MRI brain images. Here, we demonstrate the step by step process for extracting tumor objects from MRI images.

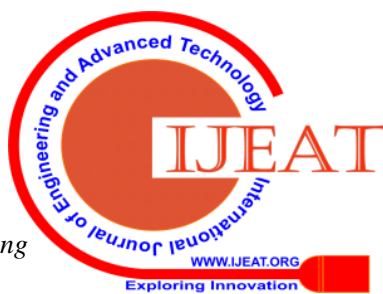




\section{Detection of Tumor Objects from MRI Brain Images using Thresholding Segmentation}

\section{Result for Normal MRI brain image:}
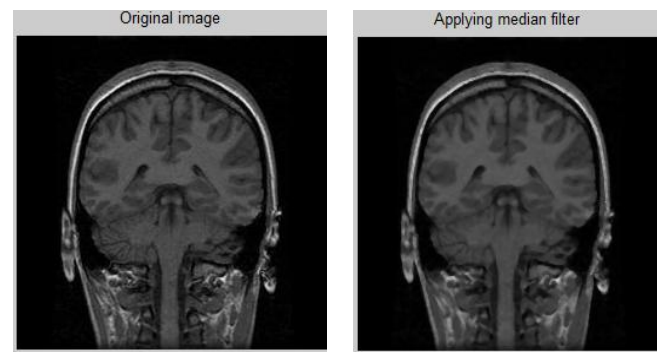

Fig. 8. Normal input MRI brain image

Fig. 9. Filtered image, Figure
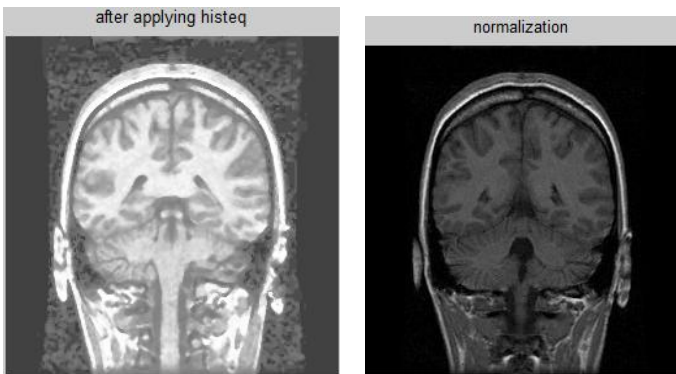

Fig. 10. Histogram Equalization output

Fig. 11. Normalization output,
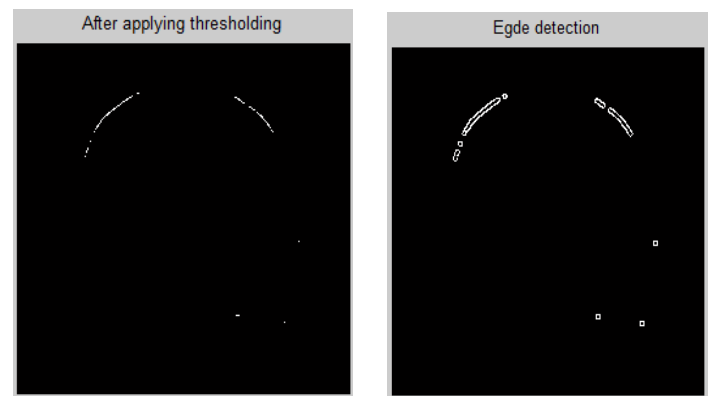

Fig. 12. Thresholding output,

Fig. 13. Canny edge detector output Filtered image,

\section{Result for abnormal MRI images}

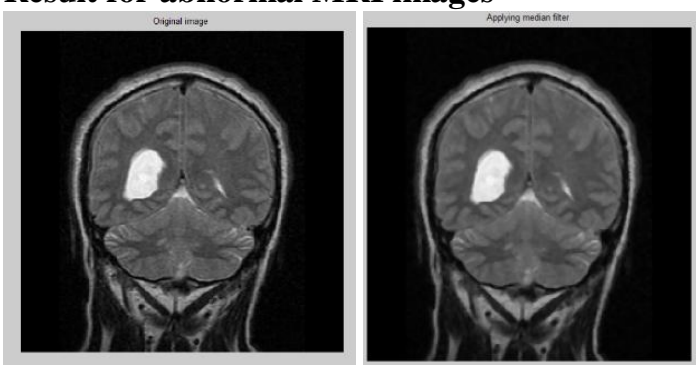

Fig. 14. Abnormal MRI image,

Fig. 15. Filtered image,
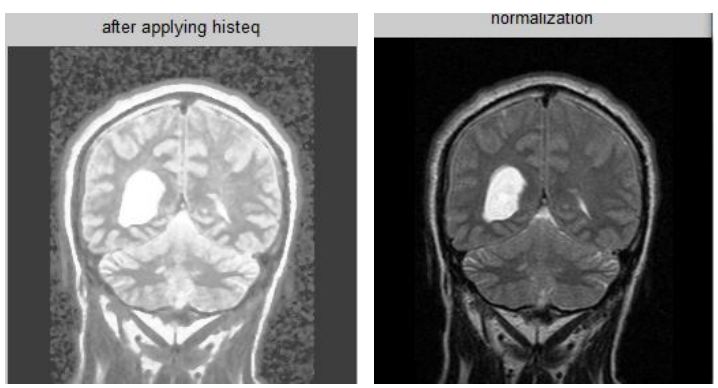

Fig. 16. Filtered image,

Fig. 17. Histogram equalization output,
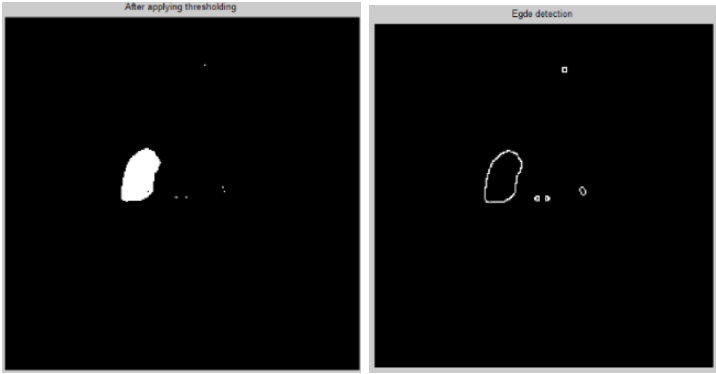

Fig. 18. Normalization output,

Fig. 19. Thresholding output, Figure (1) Canny edge detector output.

\section{Comparison:}

\section{Morphological Operation:}

From this morphological operation, the parameter values such as area, mean, standard deviation and entropy are calculated for normal and abnormal MRI image and finally tabulated the results.

The following table gives the parameter comparison results of normal and abnormal MRI brain image.

Table- I

\begin{tabular}{|l|c|l|}
\hline Parameters & $\begin{array}{c}\text { Normal Brain } \\
\text { MRI }\end{array}$ & $\begin{array}{c}\text { Abnormal Brain } \\
\text { MRI }\end{array}$ \\
\hline Entropy & 0.007638 & 0.095865 \\
\hline Area & 48.875 & 789.5694 \\
\hline Mean & 0.17843 & 3.253079 \\
\hline $\begin{array}{l}\text { Standard } \\
\text { Deviation }\end{array}$ & 5.388042 & 27.50423 \\
\hline
\end{tabular}

Table-1 Morphological comparison of normal and abnormal MRI.

Basically, these values are calculated by using the MATLAB inbuilt function. We infer from above table is the abnormal values are always high while compared with normal MRI brain image.

\section{CONCLUSION}

The proposed work presents the processing of various MRI images were taken from the MIDAS MRA and MRI-TIP brain database. The main objective of this paper is to detect the tumor objects from the MRI brain image by using thresholding segmentation. The

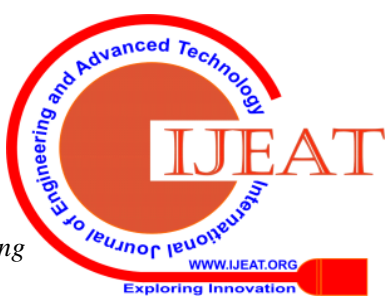


result is shown in the figures of two cases like normal and abnormal MRI brain images. The morphological operator is used to calculate the area, mean, SD and entropy. These values are calculated for the normal and abnormal case and tabulated the results. From the morphological comparison, we conclude that the abnormal parameter values are always high while compared to normal case. Finally, our proposed technique will help medical experts to easily identify the exact location of the tumor

\section{REFERENCES}

1. A.Sivaramakrishnan And Dr.M.Karnan "A Novel Based Approach For Extraction Of Brain Tumor In Mri Images Using Soft Computing Techniques", International Journal Of Advanced Research In Computer And Communication Engineering, Vol. 2, Issue 4, April 2013.

2. M. Rakesh1, T. Ravi, 2012. "Image Segmentation and Detection of Tumor Objects in MR Brain Images Using FUZZY C-MEANS (FCM) Algorithm". International Journal of Engineering Research and Applications (IJERA)

3. Anupurba Nandi, 2015. "Detection of human brain tumor using MRI image segmentation and morphological operators". IEEE International Conference on Computer Graphics, Vision and Information Security

4. Swapnil R. Telrandhe, Amit Pimpalkar, 2016. "Detection of brain tumor from MRI images by using segmentation \& SVM". World Conference on Futuristic Trends in Research and Innovation for Social Welfare W.-K. Chen, Linear Networks and Systems (Book style). Belmont, CA: Wadsworth, 1993, pp. 123-135.

5. K.Srinivas, July-2012."A Scientific Approach for Segmentation and Clustering Technique of Improved K-Means and Neural Networks". International Journal of Advanced Research in Computer Science and Software Engineering.

6. Varsha Kshirsagar*, Prof.Jagruti Panchal, 2014. "Segmentation of Brain Tumour and Its Area Calculation". International Journal of Advanced Research in Computer Science and Software Engineering Volume 4, Issue 5.

\section{AUTHORS PROFILE}

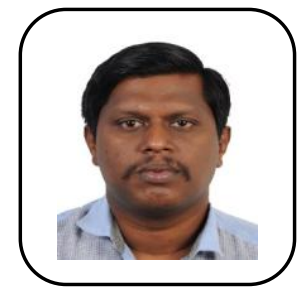

Dr. K.Sathish Kumar a Ph.D from VIT University, Vellore, India has 14 years of total teaching experience and in 9 years of his research experience he has worked in various research teams to develop new applications of evolutionary computing algorithms for solving various power system problems like unit commitment, economic dispatch, emission reduction, smart grid, power system reconfiguration and restoration. His current area of interest is studying interconnection problems in linkages of HVDC and HVAC $(765 \mathrm{kV})$ transmission lines with existing $230 \mathrm{kV}$ high voltage lines, Nano additives for high voltage XLPE cables, Optimization of smart grids. He is a member of IEEE, IEEE-PES and SSI. He has published 51 research papers in different journals and conferences of international repute and authored a book on power quality. At present he is working for School of Electrical Engineering as Associate Professor. He is the reviewer for various SCI journals like Electrical power and energy systems, ISA Transactions, AAI, etc..

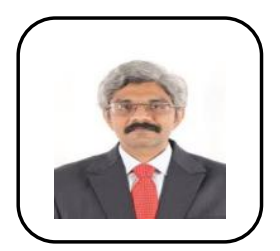

Dr .S. Venkatesh currently works at the Division of Power Systems, VIT University. S. Does research in Electrical Engineering specifically in Lightning Protection, Insulation Diagnostics, Software Engineering and Data Mining. His current project is 'PD Recognition Techniques' and "Lightning Protection System of Heritage Monuments 